\title{
Total Penectomy
}

National Cancer Institute

\section{Source}

National Cancer Institute. Total Penectomy. NCI Thesaurus. Code C159451.

Surgical removal of the entire penis. 